The Review of Economic Studies Ltd.

\author{
Implications of Efficient Risk Sharing without Commitment \\ Author(s): Narayana R. Kocherlakota \\ Source: The Review of Economic Studies, Vol. 63, No. 4 (Oct., 1996), pp. 595-609 \\ Published by: The Review of Economic Studies Ltd. \\ Stable URL: http://www.jstor.org/stable/2297795 \\ Accessed: 08/12/2010 08:25
}

Your use of the JSTOR archive indicates your acceptance of JSTOR's Terms and Conditions of Use, available at http://www.jstor.org/page/info/about/policies/terms.jsp. JSTOR's Terms and Conditions of Use provides, in part, that unless you have obtained prior permission, you may not download an entire issue of a journal or multiple copies of articles, and you may use content in the JSTOR archive only for your personal, non-commercial use.

Please contact the publisher regarding any further use of this work. Publisher contact information may be obtained at http://www.jstor.org/action/showPublisher?publisherCode=resl.

Each copy of any part of a JSTOR transmission must contain the same copyright notice that appears on the screen or printed page of such transmission.

JSTOR is a not-for-profit service that helps scholars, researchers, and students discover, use, and build upon a wide range of content in a trusted digital archive. We use information technology and tools to increase productivity and facilitate new forms of scholarship. For more information about JSTOR, please contact support@jstor.org.

The Review of Economic Studies Ltd. is collaborating with JSTOR to digitize, preserve and extend access to The Review of Economic Studies. 


\title{
Implications of Efficient Risk Sharing without Commitment
}

\author{
NARAYANA R. KOCHERLAKOTA \\ Federal Reserve Bank of Minneapolis
}

First version received January 1994; final version accepted May 1996 (Eds.)

\begin{abstract}
Consumption data generally indicates that consumption risk is not perfectly diversified across individuals. This paper considers if and when imperfect diversification is a feature of efficient allocations in a symmetric information environment without commitment. It shows that if individuals are sufficiently patient, imperfect diversification is always sub-optimal in the long run; however, if individuals are not so patient, imperfect diversification is always optimal. The paper goes on to demonstrate that the way that history matters in an efficient allocation in a symmetricinformation/no-commitment environment can be used to distinguish lack of commitment from other possible rationalizations of imperfect risk sharing, such as efficiency in the presence of asymmetric information.
\end{abstract}

One stylized description of consumption data is that, conditional on per capita consumption, individual consumption is positively correlated with current and lagged individual income. ${ }^{1}$ In a frictionless economy, this fact serves as evidence of inefficiencies in the allocation of consumption risk. However, several recent papers ${ }^{2}$ suggest that incomplete diversification of consumption risk is actually an optimal response to the technological problem that monitoring of income or effort is costly. According to this view, in any efficient allocation, consumption must be correlated with individual income in order to elicit truth-telling or adequate effort.

A problem with this justification of incomplete diversification is that within many risk-sharing pools, the degree of asymmetric information about crucial economic attributes is actually small (for example, within villages, families or even across countries). Instead, a more important informational problem is that it is costly for outside agencies to learn about the critical economic attributes of the "insiders". As Hart and Moore (1989) have emphasized, this type of informational problem makes the enforcement of contracts by third parties-that is, commitment-difficult or impossible.

The first part of this paper asks if and when the statistical properties of efficient allocations of risk in the absence of a commitment technology are consistent with incomplete diversification of individual consumption risk. It considers an infinite-horizon model with two agents who have identical preferences. Every period, the agents receive a random endowment of a perishable consumption good. Their endowments are independently and identically distributed over time. Moreover, there is no private information problem: each agent knows the current and past realizations of both endowments. Every period, the agents simultaneously transfer a non-negative amount of consumption to each other. Neither agent is able to commit to promises of future transfers.

1. See Cochrane (1991) and Townsend (1994) for empirical findings that are consistent with this description.

2. See, among others, Townsend (1987), Green (1987), Thomas and Worrall (1990), Phelan and Townsend (1991), Atkeson and Lucas (1992), and Wang (1994). 
I show formally that autarky is the worst subgame-perfect equilibrium in this infinitely repeated game, and use the results of Abreu (1988) to completely characterize the set of subgame-perfect allocations of consumption. I follow Thomas and Worrall (1988) by characterizing the Pareto frontier in the set of subgame-perfect allocations as a solution to a functional equation. This functional equation makes it simple to derive various properties about efficient allocations.

A standard folk theorem (Kimball (1988)) implies that if individuals are sufficiently patient, then some first-best allocation (that is, an allocation that is efficient in the presence of full commitment) will be subgame perfect. I prove that in this case of high patience, every efficient allocation converges monotonically with probability one to a subgameperfect first-best allocation. Hence, the long-run statistical properties of an efficient allocation without commitment look the same as those of an efficient allocation with commitment; as noted above, such statistical properties are inconsistent with data on consumption allocations.

Because of the two-sided lack of commitment, though, it is possible that no first-best allocation is subgame perfect if individuals are sufficiently impatient (even if there is a non-autarkic subgame-perfect allocation). I prove that in this case, every efficient allocation converges weakly to the same distribution of consumption, independent of initial conditions. Moreover, efficiency implies that the long-run correlation between individual consumption and current and lagged individual income, conditional on aggregate consumption, is positive. Thus, if individuals are sufficiently impatient, the statistical properties of efficient allocations in symmetric information environments without commitment are similar in important respects to those of the data.

As noted above, efficient allocations in environments with asymmetric information and full commitment also exhibit positive correlations between individual income and individual consumption. In the second part of the paper, I show how to empirically distinguish between efficient allocations in a symmetric-information/no-commitment world and efficient allocations in an asymmetric-information/full-commitment world. In particular, I demonstrate that the way history matters is very different in efficient allocations of the two types of environments.

To see how this works, suppose $M U_{t}^{n}$ is the marginal utility of consumption of agent $n$ in a given period. I show that unlike an asymmetric information environment, efficiency in a symmetric-information/no-commitment world means that the vector $\left(M U_{t}^{N} / M U_{t}^{n}\right)_{n=1}^{N-1}$ is a sufficient statistic for the future allocation of consumption. Empirically, this means that when the current consumption allocation is regressed on last period's vector $\left(M U_{t-1}^{N} / M U_{t-1}^{n}\right)_{n=1}^{N-1}$, and past realizations of consumption, the latter should be insignificant.

The above implication is testable using only consumption data (given knowledge of the marginal utility function). If both consumption and income data are available, it is possible to obtain an even stronger implication of efficiency in environments with symmetric information and no commitment. First, the consumption data can be used to determine which agents are constrained by the lack of commitment in any given period. Then efficiency dictates that the past income realizations of the constrained agents will not affect their current consumption. We can think of the economy as displaying "amnesia" because it is optimal for society to "forget" the past shocks hitting agents who become constrained. I discuss how this property can be tested using data on individual consumption and individual income. ${ }^{3}$

3. While this article was under review, Ligon, Thomas and Worrall (1996) wrote a paper which makes some of the same points as I do. In particular, they demonstrate that all efficient allocations converge to firstbest allocations if the latter are subgame perfect; also, they emphasize the "amnesia" of the optimal allocation in the two-person case (they term this property "forgiveness"). 
The theory underlying this paper is related to others in the literature. Thomas and Worrall (1988) characterize efficient allocations in an environment without commitment with one risk-averse agent and one risk-neutral agent. Haller (1990), Taub (1989), and Coate and Ravaillon (1993) characterize the conditions under which first-best allocations are subgame perfect. They discuss various sub-optimal insurance schemes when no firstbest allocation is subgame perfect, but unlike this paper, they do not talk about the properties of optimal insurance schemes. Gauthier and Poitevin (1994) explore the ability of pre-payments to improve efficiency in environments without commitment when agents have quadratic utility. Finally, Zhang (1993), Kehoe and Levine (1993), Blume and Corbae (1994), and Alvarez and Jermann (1995) investigate asset trade in environments without commitment. $^{4}$

The rest of this paper is organized as follows. Section 1 describes the basic environment. Section 2 defines and characterizes subgame perfection. Section 3 characterizes the efficient allocations (in the class of subgame-perfect allocations). Section 4 describes the long-run statistical behaviour of efficient allocations. Section 5 reports some stronger empirical implications of efficiency, and discusses how to evaluate them. Section 6 concludes.

\section{THE ENVIRONMENT}

Consider the following environment. There are two infinitely-lived agents. The state of the world in period $t$ is stochastic and is determined by the realization of a discrete i.i.d. random variable $\theta_{t}$, with support equal to $\{1,2,3, \ldots, S\}$; the probability of $\theta_{t}$ equalling $s$ is denoted by $\pi_{s}$, where $\pi_{s}>0$ for all $s$. There is a single perishable consumption good. The endowments of the two agents in period $\mathrm{t},\left(y_{t}^{1}, y_{t}^{2}\right)$, are determined by the realization of $\theta$ in that period; the aggregate endowment $\left(y_{t}^{1}+y_{t}^{2}\right)$ is denoted by $Y_{t}$. I assume that the joint distribution of the endowments is symmetric in the sense that the probability of occurrence of $\left(y, y^{\prime}\right)$ is the same as the probability of occurrence of $\left(y^{\prime}, y\right)$; I also assume that $Y_{t}$ is positive with probability one. (Note that this description of the environment allows $y^{1}$ and $y^{2}$ to be independent without restricting attention to that case.)

An allocation is a non-negative stochastic vector process $\left(\left(c_{t}^{j}\right)_{t=1}^{\infty}\right)_{j=1}^{2}$ which is measurable with respect to current and past realizations of $\theta_{t}$. A feasible allocation is a process such that $c_{t}^{1}+c_{t}^{2} \leqq Y_{t}$. In period $t$, the two agents have identical preferences described by the utility function:

$$
E_{t} \sum_{\tau=0}^{\infty} \beta^{\tau} u\left(c_{t+\tau}\right), \quad 0<\beta<1
$$

The cardinal utility function $u$ is increasing and strictly concave and continuously differentiable. To guarantee an interior solution, I further assume that $\lim _{c \rightarrow 0} u^{\prime}(c)=\infty$. (When I refer to the utility derived by agent $j$ from a given allocation, I mean his ex ante utility, which is evaluated before any uncertainty has been resolved.)

It is natural in this environment to use the following definition of first-best.

Definition 1.1. An allocation is first best if $c_{t}^{1}+c_{t}^{2}=Y_{t}$ for all dates and states and $u^{\prime}\left(c_{t}^{1}\right) / u^{\prime}\left(c_{t}^{2}\right)$ is constant over all dates and states.

4. Several other papers examine the properties of efficient allocations in environments with one-sided commitment-e.g. Atkeson (1991), Marcet and Marimon (1992), and Phelan (1995). Marcet and Marimon (1995) present a general procedure to numerically solve for efficient allocations in a wide range of environments with private information and imperfect commitment. 
It is well-known that in any first-best allocation, each individual's consumption is a timeand state-invariant function of the aggregate endowment. Note that splitting the aggregate endowment in half at every date and in every state is a first-best allocation; also, in any other first-best allocation, one individual receives less than half the aggregate endowment at every date and in every state.

\section{SUBGAME-PERFECT ALLOCATIONS}

The two agents interact in the following way. At the beginning of period $t$, the realization of $\theta_{t}$ becomes known to both of them. At that point, each of the two individuals simultaneously transfers a non-negative amount of his current income to the other individual (note that this transfer may be zero). Thus, a period- $t$ history in this dynamic game is a sequence of realizations for $\theta_{t}$ and non-negative transfers made by the agents:

$$
\left(\theta_{1}, T R_{1}, \theta_{2}, T R_{2}, \ldots, T R_{t-1}, \theta_{t}\right) .
$$

A strategy for an agent specifies his action after each possible history; thus, in period $t$, agent $j$ 's strategy is a mapping from possible histories into transfer amounts.

A subgame-perfect equilibrium specifies a strategy for each agent such that a player's action (that is, choice of transfer amounts) at a given history is optimal given the other player's strategy. A subgame-perfect equilibrium allocation is the consumption process (feasible by construction) that results from the implementation of these strategies.

It is easy to characterize the set of subgame-perfect allocations using the techniques of Abreu (1988). We first identify the subgame-perfect allocation that provides the least utility to both of the agents.

Lemma 2.1. The autarkic allocation, $c_{t}^{j}=y_{t}^{j}$ for $j=1,2$ and all $t$, is subgame perfect and provides less utility to both agents than any other subgame-perfect allocation.

Proof. See Appendix.

In what follows, I will use $V_{\text {aut }}$ to denote the utility derived by any agent in autarky; note that the symmetry of the joint distribution of the aggregate endowments guarantees that $V_{\text {aut }}$ is the same for all individuals.

Since autarky provides less utility than any other subgame-perfect allocation, it is the worst possible punishment that can be provided in this environment for an agent who deviates from a proposed allocation. This intuition allows us to deduce Proposition 2.1.

Proposition 2.1. A feasible allocation is subgame-perfect if and only if it satisfies:

$$
u\left(c_{t}^{j}\right)+E_{t} \sum_{\tau=1}^{\infty} \beta^{\tau} u\left(c_{t+\tau}^{j}\right) \geqq u\left(y_{t}^{j}\right)+\beta V_{\text {aut }}, \quad j=1,2
$$

for all dates and states.

\section{Proof. See Appendix.}

This proposition uses the techniques of Abreu (1988) to demonstrate that the set of subgame-perfect allocations (which I will henceforth label $\Gamma$ ) can be fully characterized using some simple constraints. In particular, note that (1) and (2) imply that $\Gamma$ is compact in the product topology and that $\Gamma$ is convex. Throughout the rest of the paper, $I$ assume 
that the specification of the joint distribution of the individual endowments, $\beta$ and the utility function $u$ are such that there exists some non-autarkic subgame-perfect allocation. ${ }^{5}$

It is well-known that a first-best allocation may be subgame perfect if $\beta$ is sufficiently large (see Kimball (1988)). For all values of $\beta$, though, there exists some first-best allocation that is not subgame perfect, even though both agents get more utility from the allocation than they do in autarky. Indeed, if $\beta$ is small enough, then there may not be any subgame-perfect first-best allocations. ${ }^{6}$

\section{EFFICIENT ALLOCATIONS}

I define an efficient allocation as follows. ${ }^{7}$

Definition 3.1. An allocation $\left(c^{1}, c^{2}\right)$ in $\Gamma$ is efficient if there exists no other element in $\Gamma$ that provides both individuals with at least as much utility and one of them with more.

Define $V_{\max }$ to be the maximal level of utility available to a given agent from an allocation in $\Gamma$, and define the function $V:\left[V_{\text {aut }}, V_{\max }\right] \rightarrow\left[V_{\text {aut }}, V_{\max }\right]$ by:

$$
\begin{array}{cl}
V\left(u_{0}\right)=\operatorname{Max}_{\left(c^{1}, c^{2}\right)} & E_{0} \sum_{t=1}^{\infty} \beta^{t-1} u\left(c_{t}^{2}\right) \\
\text { s.t. } & \left(c^{1}, c^{2}\right) \in \Gamma \\
\text { s.t. } & E_{0} \sum_{t=1}^{\infty} \beta^{t-1} u\left(c_{t}^{1}\right) \geqq u_{0} .
\end{array}
$$

The function $V$ represents the Pareto frontier in this environment. Using arguments analogous to those of Thomas and Worrall (1988), we can conclude that $V$ is differentiable and that $V$ satisfies the following functional equation:

$$
\begin{array}{rll}
V\left(u_{0}\right)= & \operatorname{Max}_{\left(c_{s}, u_{s}\right) f} & \sum_{s=1}^{S} \pi_{s}\left[u\left(Y_{s}-c_{s}\right)+\beta V\left(u_{s}\right)\right] \\
\text { s.t. } & \sum_{s=1}^{S} \pi_{s}\left[u\left(c_{s}\right)+\beta u_{s}\right]=u_{0} . \\
\text { s.t. } & u\left(c_{s}\right)+\beta u_{s} \geqq u\left(y_{s}^{1}\right)+\beta V_{\text {aut }}, \quad \text { for all } s . \\
\text { s.t. } & u\left(Y_{s}-c_{s}\right)+\beta V\left(u_{s}\right) \geqq u\left(Y_{s}-y_{s}^{1}\right)+\beta V_{\text {aut }}, \quad \text { for all } s . \\
\text { s.t. } & u_{s} \in\left[V_{\text {aut }}, V_{\max }\right] .
\end{array}
$$

This characterization shows that we can think about the construction of efficient subgameperfect allocations using the following mechanical metaphor. A social planner enters period $t$ having promised agent 1 a certain amount of ex ante utility, $u_{0}$. Taking this promise as

5. A sufficient condition for the existence of a non-autarkic subgame-perfect allocation is that there is some state $s$ with probability of occurrence $\pi \leqq 0.5$ and:

$$
\{(1-\beta)+\beta \pi\} u^{\prime}\left(y_{s}^{1}\right)-\beta \pi u^{\prime}\left(y_{s}^{2}\right)<0 .
$$

(See Kocherlakota (1994) for a proof.)

6. Because any asymmetric first-best allocation makes some agent strictly worse off than the symmetric first-best allocation, there is a subgame-perfect first-best allocation if and only if the symmetric first-best allocation is subgame perfect. The symmetric first-best allocation is subgame perfect if:

$$
\sum_{r=1}^{S} \pi_{r} u\left(Y_{r} / 2\right) /(1-\beta) \geqq u\left(y_{s}\right)+\beta V_{\text {aut }}
$$

for all $s$.

7. In this particular environment, any efficient subgame-perfect equilibrium is strongly renegotiation-proof in the sense of Farrell and Maskin (1989): see Asheim and Strand (1991). 
given in the form of (P1), the planner seeks to maximize the amount of ex ante utility agent 2 receives. The planner determines how much consumption to give to or take from agent 1 and how much future utility to promise agent 1 , contingent on each state of the world. He must take into account the sustainability constraints (P2)-(P3) that capture his inability to force the agents to give up consumption beyond threatening them with future autarky. The last constraint on $u_{s}$ requires the choices of the social planner to lie in the domain of the value function $V$.

Now, consider an efficient allocation which provides agent 1 with utility equal to $u_{0}$. The maximization problem in (FE) makes clear that we can divide the possible states of the world in period one into three groups.

$S_{1}$ : states in which constraint (P2) binds.

$S_{2}$ : states in which constraint (P3) binds.

$S_{3}$ : states in which neither constraint binds.

(By "binds" I mean that the multiplier on the constraint is positive.) It is easy to see that the intersection of $S_{1}$ and $S_{2}$ must be empty. Suppose $s$ lies in $S_{1}$. Then, $c_{s} \leqq \mathrm{y}_{s}^{1}$ because $u_{s} \geqq V_{\text {aut }}$. If $s$ lies in $S_{2}$, then $c_{s} \geqq y_{s}^{1}$. It follows that if $s$ lies in $S_{1}$ and $S_{2}$, then $c_{s}=y_{s}^{1}$ and $u_{s}=V_{\text {aut }}$ and $V\left(u_{s}\right)=V_{\text {aut }}$. But this means that $V\left(V_{\text {aut }}\right)=V_{\text {aut }}$, which is impossible as long as there exists some subgame-perfect allocation that is non-autarkic.

The first-order conditions with respect to $u_{s}$ in the maximization problem in (FE) take the following form:

$$
\begin{aligned}
\beta \pi_{s} V^{\prime}\left(u_{s}\right)+\lambda \beta \pi_{s}+\beta \mu_{s}+v_{s} \beta V^{\prime}\left(u_{s}\right)=0 & \text { if } u_{s} \in\left(V_{\text {aut }}, V_{\max }\right) \\
\leqq 0 & \text { if } u_{s}=V_{\text {aut }} \\
\geqq 0 & \text { if } u_{s}=V_{\max }
\end{aligned}
$$

where $\lambda$ is the multiplier on (P1), $\mu_{s}$ is the multiplier on (P2), and $v_{s}$ is the multiplier on (P3). We can use these first-order conditions to describe the evolution of ex ante utility over time. In particular, the envelope theorem tells us that $\lambda=-V^{\prime}\left(u_{0}\right)$. Hence, if $s$ lies in $S_{1}$, and $u_{0}<V_{\max }$, then $V^{\prime}\left(u_{s}\right)<V^{\prime}\left(u_{0}\right)$; the strict concavity of $V$ then implies that $u_{s}>u_{0}$. On the other hand, if $s$ lies in $S_{2}$ and $u_{0}>V_{\text {aut }}$, then $V^{\prime}\left(u_{s}\right)>V^{\prime}\left(u_{0}\right)$ and $u_{s}<u_{0}$. In words, this analysis tells us that it is efficient to induce an agent with a binding sustainability constraint to provide consumption today by promising him more utility in the future. ${ }^{8}$ In contrast, if $s$ lies in $S_{3}$, then $\mu_{s}$ and $v_{s}$ are both zero, and so $V^{\prime}\left(u_{s}\right)=V^{\prime}\left(u_{0}\right)$; in other words, $u_{s}=u_{0}$.

The first-order conditions with respect to $c_{s}$ are:

$$
-\pi_{s} u^{\prime}\left(Y_{s}-c_{s}\right)+\lambda \pi_{s} u^{\prime}\left(c_{s}\right)+\mu_{s} u^{\prime}\left(c_{s}\right)-v_{s} u^{\prime}\left(Y_{s}-c_{s}\right)=0 .
$$

Combining the two sets of first-order conditions, we obtain:

$$
\begin{array}{rlrl}
u^{\prime}\left(Y_{s}-c_{s}\right) / u^{\prime}\left(c_{s}\right) & =-V^{\prime}\left(u_{s}\right) & & \text { if } u_{s} \in\left(V_{\text {aut }}, V_{\max }\right) \\
& \geqq-V^{\prime}\left(V_{\max }\right) & \text { if } u_{s}=V_{\max } \\
& \leqq-V^{\prime}\left(V_{\text {aut }}\right) & & \text { if } u_{s}=V_{\text {aut }} .
\end{array}
$$

This first-order condition is valid in every date and state. It implies that given a specification of $Y_{s}, u_{s}$ is a non-decreasing function of $c_{s}$. This tells us that given a group of states with

8. If $u_{0}=V_{\text {aut }}$, and $s$ lies in $S_{2}$, then $u_{s}=v_{\text {aut }}$; similarly, if $u_{0}=V_{\max }$, and $s$ lies in $S_{1}$, then $u_{s}=v_{\max }$. 
the same aggregate $Y$, a state $s$ lies in $S_{1}$ if $y_{s}^{1}$ is high and $s$ lies in $S_{2}$ if $y_{s}^{2}$ is high: agents tend to face binding sustainability constraints when their income shocks are high.

We can use these first-order conditions to deduce the following contemporaneous interaction between agent 1's consumption and agent 1's income, conditional on a particular realization of the aggregate endowment and on the history of past shocks (as summarized by $u_{0}$ ). When agent 1 's income is low, then agent 2 's sustainability constraint is binding; as agent 2's income realization increases, he requires a larger "bribe" in terms of consumption (and future utility) to keep him in the optimal allocation. When agent 1's income is about average, then neither sustainability constraint is binding: in that region, his consumption is flat. When agent 1's income is high, his sustainability constraint binds and his consumption is positively correlated with income. Thus, as long as $S_{1}$ or $S_{2}$ is non-empty, there is a positive contemporaneous correlation between individual income and individual consumption.

As Thomas and Worrall (1988) emphasize, though, the interesting feature about lack of commitment is that it introduces persistence into the efficient allocations even if there are no exogenous dynamics. When an agent receives a sufficiently high income shock so that his sustainability constraint is binding, (1) implies that $u_{s}$ rises above $u_{0}$. This increase in $u_{s}$ will alter the allocation of consumption in the following period. Thus, the realization of income in period $t$ has an effect on consumption in period $(t+1)$. Intuitively, it is optimal for any shock that cannot be smoothed over states because of limited enforcement to be smoothed over time; this gives rise to persistence in individual consumption.

The relationship between individual consumption and current and lagged individual income gives rise to the following proposition.

Proposition 3.1. In an efficient allocation, $\operatorname{Cov}\left(c_{t}^{1}, y_{t-k}^{1} \mid Y_{t-k}\right) \geqq 0$ for $k \geqq 0$. For $k=$ 0 , if this conditional covariance is zero for all realizations of $Y_{t}$, then the efficient allocation is first best in all ensuing dates and states.

Proof. See Appendix.

Hence, in any efficient allocation, the correlation between individual consumption and current and lagged individual income, conditional on aggregate consumption, is nonnegative. The next section investigates under what conditions, efficiency in the face of limited enforcement implies that this conditional correlation is positive, as is found in the data.

\section{THE LONG-RUN BEHAVIOUR OF EFFICIENT ALLOCATIONS}

In this section, I examine the long-run dynamics of efficient allocations. I will focus on the long-run behaviour of the ex ante utility received by agent $1, u_{t} \equiv$ $E_{t} \sum_{s=0}^{\infty} \beta^{s} u\left(c_{t+s+1}\right)$, although the results are easily generalized to consumption itself.

\section{A. When Subgame-Perfect First-Best Allocations Exist}

Suppose the symmetric first-best allocation is subgame perfect. Define $u_{\mathrm{FB}}$ to be the lowest level of ex ante utility associated with a subgame-perfect first-best allocation, and $u^{\mathrm{FB}}$ to be the highest level of ex ante utility associated with a subgame-perfect first-best allocation. 
Similarly, define the stochastic process $c^{\mathrm{FB}}$ to be individual l's allocation in the first-best allocation that provides him ex ante utility $u^{\mathrm{FB}}$, and define $c_{\mathrm{FB}}$ to be individual l's consumption stream in the first-best allocation that provides him ex ante utility $u_{\mathrm{FB}}$. Note that the definition of $u_{\mathrm{FB}}$ guarantees that if agent $j$ is promised more than $u_{\mathrm{FB}}$ in any given state, then his sustainability constraint does not bind in that state.

Suppose $u_{0}$ lies in the set $I=\left[u_{\mathrm{FB}}, u^{\mathrm{FB}}\right]$. Then there exists a subgame-perfect first-best allocation which provides this ex ante utility to agent 1 ; this allocation is therefore efficient. In these kinds of allocations, $u_{t}$ is constant over all dates and states at its initial level $u_{0}$.

It is more interesting to think about the dynamic path of $u_{t}$ in efficient allocations in which $u_{0}$ does not lie in $I$. Suppose for example $u_{0}>u^{\mathrm{FB}}$. Agent 1 's utility can only rise above $u_{0}$ in period 1 if his sustainability constraint binds; but, because $u_{0}>u_{\mathrm{FB}}$, agent 1's constraint can never bind if he is promised more than $u_{0}$. It follows that if $u_{0}>u^{\mathrm{FB}}$, agent l's sustainability constraint never binds, and with probability one, $u_{1} \leqq u_{0}$.

We also know that if $u_{0}>u^{\mathrm{FB}}$, agent 2's sustainability constraint binds with positive probability in period 1; otherwise, neither agent's sustainability constraint is binding in any state, and $u_{0}$ would be associated with a subgame-perfect first-best allocation. So, if $u_{0}>u^{\mathrm{FB}}$, agent 1 's utility declines with positive probability. Can agent 1's utility ever decline below $u^{\mathrm{FB}}$ ? The answer is no. Agent 2's utility can only rise above his initial level $V\left(u_{0}\right)$ in any state if his sustainability constraint is binding in that state; but his constraint cannot bind in any state in which he is promised more than $u_{\mathrm{FB}}$ (and agent 1 is promised less than $u^{\mathrm{FB}}$ ).

Thus, if $u_{0}>u^{\mathrm{FB}}$, there is some state in period 1 such that agent 2's utility is no more than $u_{\mathrm{FB}}$, and agent 1's utility is strictly less than $u_{0}$. Reasoning by induction, this means that in any efficient allocation such that $u_{0}>u^{\mathrm{FB}}, u_{t}$ either equals $u_{t-1}$ or falls below $u_{t-1}$. Eventually, $u_{t}$ converges to $u^{\mathrm{FB}}$.

This logic generates the following proposition.

Proposition 4.1. Suppose that some first-best allocation is subgame perfect. If $u_{0}>u^{\mathrm{FB}}$, then with probability one, $u_{t}$ converges monotonically to $u^{\mathrm{FB}}$. If $u_{0}<u_{\mathrm{FB}}$, then with probability one, $u_{t}$ converges monotonically to $u_{\mathrm{FB}}$.

\section{Proof. See Appendix.}

The above result can be extended to consumption: in an efficient allocation, along almost every sample path, $\left|c_{t}-c^{\mathrm{FB}}\left(Y_{t}\right)\right|$ converges to 0 (where $c^{\mathrm{FB}}\left(Y_{t}\right)$ is the amount of consumption that the least equal subgame-perfect first-best allocation delivers to agent 1 when the aggregate endowment equals $Y_{t}$ ). In this sense, if a first-best allocation is subgame perfect, then any efficient allocation of consumption converges with probability one to a first-best allocation. Thus, if people are sufficiently patient that some first-best allocation is subgame perfect, then absence of commitment cannot justify the observed lack of diversification in individual consumption as being efficient.

\section{B. When No First-Best Allocations Are Subgame Perfect}

Suppose that the contemporaneous covariance between individual income and individual consumption, conditional on aggregate consumption, is zero in an efficient allocation. The second part of Proposition 3.1 then implies that the ensuing allocation of resources in all future dates and states is first best. Thus, if no first-best allocation is subgame perfect, the 
conditional covariance is positive in any efficient allocation. In this subsection, I strengthen this result considerably, and show that in the long run, not just the sign but the magnitude of this correlation is the same in all efficient allocations. From an empirical point of view, this is a crucial result: it means that the quantitative implications of efficiency can be compared to the data without having to know (the generally unobservable) initial division of surplus between the agents.

To obtain this limiting result, it is helpful to first note from the form of the functional equation (FE) that in an efficient allocation, $u_{t}$ must follow a Markov process with realizations in the compact set $\left[V_{\text {aut }}, V_{\max }\right]$. In particular, given an efficient allocation:

$$
\operatorname{Pr}\left(u_{t+1}=u \mid u_{t}, u_{t-1}, \ldots, u_{0}\right)=\operatorname{Pr}\left(u_{t+1}=u \mid u_{t}\right) .
$$

The easily verified continuity and monotonicity of the policy functions $u_{s}$ are enough to insure that this Markov process is monotone and satisfies the Feller property (see Stokey and Lucas with Prescott (SLP) (1989)); this tells us that for any initial $u_{0}$, the utility Markov process converges weakly to some stationary distribution. However, we have to guarantee that utility in different efficient allocations (that is, different initial conditions for $u_{0}$ ) do not converge to different limiting distributions. The following proposition delivers this guarantee by demonstrating that the utility Markov process satisfies a crucial mixing condition.

Proposition 4.2. Suppose there is no subgame-perfect first-best allocation. Then, as $t$ goes to infinity, $\operatorname{Pr}\left(u_{t} \mid u_{0}\right)$ converges weakly to the same non-degenerate limiting distribution for all $u_{0}$ in $\left[V_{\text {aut }}, V_{\max }\right]$.

\section{Proof. See Appendix.}

It is immediate from Proposition 4.2 that the limiting joint distribution of the agents' consumptions exists and is the same in all efficient allocations.

\section{THE EFFECT OF HISTORY IN EFFICIENT ALLOCATIONS}

Wang (1994) looks at the properties of the efficient allocations of risk in an environment in which two agents cannot observe the realizations of each other's endowments but can fully commit to future transfer arrangements. He finds that in the long run, the unconditional probability distribution of consumption converges to a limit that is independent of the initial division of surplus between the two agents. (Wang's results in this regard differ from much of the rest of the private information literature (e.g. Atkeson and Lucas (1992)), because, as I do in this paper, he requires individual consumption sets to be bounded from above and below.) He proves that in efficient allocations, individual consumption is positively correlated with individual income, conditional on the realization of aggregate income, and that individual consumption is potentially related to lagged realizations of both individuals' incomes.

Thus, the implications of efficiency in symmetric-information/no-commitment worlds (with no subgame-perfect first-best allocation) are qualitatively similar to the implications of efficiency in asymmetric-information/full-commitment environments. Given this similarity, it is natural to ask how to determine which of the two models is more empirically relevant. As it turns out, the way history matters in efficient allocations in the two environments is quite different. 
To see this, note that the functional equation (FE) implies that in an efficient allocation in a symmetric-information/no-commitment world, the past is fully summarized by the current position of the economy on the Pareto frontier $\left(u_{t}\right)$. If agent 1 's ex ante utility were observable, then this implication would be testable, but in general, there is little data available on individual ex ante utilities. However, we have seen in (2) that efficiency implies that:

$$
u^{\prime}\left(c_{t}^{2}\right) / u^{\prime}\left(c_{t}^{1}\right)=-V^{\prime}\left(u_{t}\right) \text { if } u_{t} \in\left(V_{\text {aut }}, V_{\max }\right)
$$

where $c_{r}^{j}$ is agent $j$ 's consumption in period $t$. This means that there is a one-to-one relationship between the ratio $u^{\prime}\left(c_{t}^{2}\right) / u^{\prime}\left(c_{t}^{1}\right)$ and the position of the economy on the Pareto frontier. Hence, in a symmetric-information/no-commitment world, the marginal utility ratio $u^{\prime}\left(c_{t}^{2}\right) / u^{\prime}\left(c_{t}^{1}\right)$ is a sufficient statistic for the evolution of efficient allocations.

The logic of the result extends easily to $N$-person symmetric-information/no-commitment environments. Define $\gamma_{1}$ to be an $(N-1)$-dimensional vector where the $n$th component $\gamma_{t}^{n}$ is equal to the ratio $u^{\prime}\left(c_{t}^{N}\right) / u^{\prime}\left(c_{t}^{n}\right)$; define $V\left(u^{1}, \ldots, u^{N-1}\right)$ to be the maximal level of utility that agent $N$ can get from a subgame-perfect allocation, given that agent $n, n<N$, gets utility level $u^{n}$. Then, the first-order conditions of an analogue to (FE) imply that if the utility vector $\left(u_{t}^{n}\right)_{n=1}^{N-1}$ is in the interior of the domain ${ }^{9}$ of the value function $V$, then the gradient of $V$ equals $-\gamma_{t}$. Because $V$ is strictly concave, knowing the gradient is equivalent to knowing the position on the Pareto frontier; as in the two-agent case, $\gamma_{r}$ is a sufficient statistic for the evolution of the system over time. ${ }^{10}$

This implication of efficiency in symmetric-information/no-commitment environments can be evaluated using a panel of $T$ observations over time on the consumptions of $N$ people in a risk-sharing pool. " The sufficiency of $\gamma_{t}$ as a summary statistic for the past can be written formally as:

$$
\operatorname{Pr}\left(\left(c_{t+1}^{n}\right)_{n=1}^{N} \mid \gamma_{t}, Y_{t},\left(\left(c_{t-s}^{n}\right)_{n=1}^{N}\right)_{s=1}^{t}\right)=\operatorname{Pr}\left(\left(c_{t+1}^{n}\right)_{n=1}^{N} \mid \gamma_{t}\right)
$$

Thus, the sufficiency of $\gamma_{t}$ means that current and lagged consumption have no additional explanatory power beyond $\gamma_{t}$ in forming forecasts of future consumption. One way to test this restriction is to run a time-series regression of $c_{t+1}$ (keeping $n$ fixed) on the lagged vector $\gamma_{t}$ and on other lagged information; in this regression, the other lagged information should be insignificant.

It is also true that the current position of the economy on the Pareto frontier is a sufficient statistic for the future evolution of efficient allocations in economies with private information and full commitment. However, in a private information world, consumption in any given state enters into the incentive compatibility constraints for other states; consequently, there is no direct linkage between the current realization of the vector $\gamma_{t}$ of marginal utility ratios and the current realization of the slope of the Pareto frontier. It follows that the vector of marginal utility ratios will typically not be a sufficient statistic for the past in efficient allocations if information is private.

9. In a two-person allocation problem, the domain of the value function is simply the interval [ $\left.V_{\text {aut }}, V_{\max }\right]$. In an $N$-person allocation problem, the $(N-1)$-dimensional domain of the value function is still convex, but the set is not a cross-product of intervals.

10. As stated, this result relies on the i.i.d. structure of individual incomes. More generally, if the state $\theta_{t}$ is first-order Markov, then efficiency implies that the vector $\left(\gamma_{t}, \theta_{t}\right)$ is a sufficient statistic for the evolution of the system.

11. Throughout the above discussion, I assume that the econometrician knows a priori the cardinal utility function $u$. This assumption is certainly not true in all settings. However, if $u$ is known at least up to a finitedimensional parameter, the restrictions implied by efficiency should serve to identify the unknown parameters (much as the restrictions implied by individual optimality do so in tests of asset-pricing models). Also, if $u(c)=$ $c^{1-\alpha} /(1-\alpha)$, then the parameter $\alpha$ is irrelevant for the tests discussed in the text. 
Thus, we can use consumption data to discriminate between two competing hypotheses about the sources of imperfect consumption diversification. However, it is still true

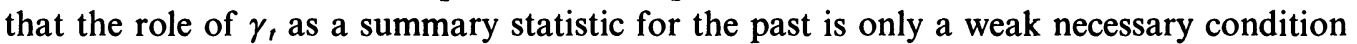
of efficiency in symmetric-information/full-commitment environments; many inefficient allocations also satisfy this property. For example, if $\left(c^{1}, c^{2}\right)$ is the equilibrium allocation that emerges from two individuals trading a single risk-free security over time, the marginal utility ratio is a sufficient statistic to describe the evolution of the allocation. Nonetheless, the allocation is typically inefficient. ${ }^{12}$

Fortunately, the econometrician can test stronger implications of efficiency if he has data on individual income as well as individual consumption. To obtain these stronger implications of efficiency, it is first necessary to sort the $N$ people in any given period into those who are constrained by their sustainability constraint and those who are not. Suppose first that agent $N$ is unconstrained in period $t$ and agent $n$ is constrained. Just as in the twoperson case, agent $n$ 's binding sustainability constraint implies that the partial derivative of the value function $V$ with respect to $u_{t}^{n}$ must be lower in period $t$ than it was last period; hence, $\gamma_{t}^{n}>\gamma_{t-1}^{n}$. (Recall that the negative of the gradient of $V$ equals the vector $\gamma_{t}$ with $n$th component $\gamma_{t}^{n}=u^{\prime}\left(c_{t}^{N}\right) / u^{\prime}\left(c_{t}^{n}\right)$ if the vector of reservation utilities lies in the interior of the domain of $V$.)

Reasoning in a similar way, we see that if agent $N$ is unconstrained and agent $n$ is unconstrained, then $\gamma_{t}^{n}=\gamma_{t-1}^{n}$. On the other hand, if agent $N$ is constrained, and agent $n$ is unconstrained, then $\gamma_{t}^{n}<\gamma_{t-1}^{n}$; if agent $N$ is constrained, and both agents $n$ and $m$ are unconstrained, then $\gamma_{t}^{n} / \gamma_{t}^{m}=\gamma_{t-1}^{n} / \gamma_{t-1}^{m}$. We can combine all of this analysis to find the set $C_{t}$ of constrained agents and the set $U_{t}$ of unconstrained agents in any date and state using data on their consumptions. Define:

$$
R_{r} \equiv \min _{n} \gamma_{r}^{n} / \gamma_{t-1}^{n}
$$

Then:

$$
\begin{gathered}
R_{t}>1 \Rightarrow C_{t}=\{1,2, \ldots, N-1\} \quad \text { and } U_{t}=\{N\} . \\
R_{t}=1 \Rightarrow C_{t}=\left\{n \mid \gamma_{t}^{n}>\gamma_{t-1}^{n}\right\} \text { and } U_{t}=\left\{n \mid \gamma_{t}^{n}=\gamma_{t-1}^{n}\right\} . \\
R_{t}<1 \Rightarrow C_{t}=\left\{n \mid \gamma_{t}^{n} / \gamma_{t-1}^{n}>R_{t}\right\} \cup\{N\} \text { and } U_{t}=\left\{n \mid \gamma_{t}^{n} / \gamma_{t-1}^{n}=R_{t}\right\} .
\end{gathered}
$$

Our earlier analysis about how history matters in an efficient allocation showed that the current realization of $\gamma_{t}$ is determined wholly by the lagged realization $\gamma_{t-1}$ and the current realization of individual incomes. Put another way, there is a function $g$ such that:

$$
\left(c_{t}^{n}\right)_{n=1}^{N}=g\left(\gamma_{t-1},\left(y_{t}^{n}\right)_{n=1}^{N}\right) .
$$

But, as it turns out, efficiency restricts the form of this function. Suppose an agent is promised ex ante utility $u_{t-1}^{n}$ before the resolution of uncertainty in period $t$. He then gets a sufficiently high income realization that he hits his sustainability constraint in period $t$. Given that this occurs, when the planner chooses a new level of ex ante utility for agent $n$, the choice ignores the past promise $u_{t-1}^{n}$; all that matters is that the agent is currently bound by a sustainability constraint-the level of which is determined wholly by $y_{t}^{n}$.

12. The work of Beaudry and DiNardo (1995) is susceptible to this criticism: they test implications of optimal labour contracting in the absence of commitment that are also characteristic of a wide class of suboptimal contracts. 
This kind of reasoning tells us that in an efficient allocation, there is a function $h$ such that:

$$
c_{t}^{n}=h\left(y_{t}^{n},\left(\gamma_{t-1}^{m}, y_{t}^{m}\right)_{m \in U_{t}}\right)
$$

for any $\boldsymbol{n}$ in the set of constrained agents $C_{t}$. (Recall that $U_{t}$ is the set of unconstrained agents.) Note that $h$ is symmetric in $\left(\gamma_{t-1}^{m}\right)_{m \in U_{t}}$ and symmetric in $\left(y_{t}^{m}\right)_{m \in U_{t}}$. The key difference between (3) and (4) is that (4) requires $c_{t}^{n}$ not to depend on the value of $\gamma_{t-1}^{n}$, once it is known that agent $n$ is constrained. The evolution of efficient allocations displays "amnesia" in the sense that the planner essentially "forgets" the past income realizations, as summarized through $\gamma_{t-1}^{n}$, of any agents who are constrained in period $t$.

Empirically, this implication can be tested by regressing all $c_{t}^{n}$ for which $n$ lies in $C_{t}$ on the corresponding variables $\left(\gamma_{t-1}^{n}, y_{t}^{n},\left(\gamma_{t-1}^{m}, y_{t}^{m}\right)_{m \in U_{t}}\right)$. (Note that this regression implicitly exploits the stability of $h$ both over time and across all constrained individuals.) Amnesia implies that in this regression, the variable $\gamma_{t-1}^{n}$ should be insignificant.

All of the above analysis assumes that the econometrician has access to well-measured consumption and income data. In many situations, this assumption is troubling. Figuring out how to evaluate these types of empirical implications in the face of measurement error is an interesting problem, but one that is beyond the scope of this paper.

\section{CONCLUSIONS}

The main message of this paper is that at least in a qualitative sense, a model with two-sided lack of commitment, symmetric information and no subgame-perfect first-best allocations is capable of generating implications that are consistent with casual characterizations of individual and cross-country data on consumption and income. For example, in stochastic steady state, there is idiosyncratic risk remaining in individual consumption, and individual consumption is (potentially) conditionally correlated with many lags of income.

To contrast the "efficiency without commitment" explanation from other rationalizations of these stylized facts about consumption, the paper shows that history matters in a distinctive manner in efficient allocations in symmetric-information/no-commitment environments. In particular, in the evolution of efficient allocations over time, the (normalized) vector of marginal utilities is a sufficient statistic for all past information, and that the economy "forgets" the past realizations of income for agents who are currently constrained.

\section{APPENDIX}

Proof of Lemma 2.1. It is clear that autarky is subgame perfect. Now consider the set of possible periodzero utility levels that agent 1 derives from subgame-perfect allocations. This set is bounded from below (by 0 ). Hence, it has an infimum; call this value $M$.

Consider an arbitrary subgame-perfect allocation $(c$,$) , and let V_{0}$ denote the utility derived by agent 1 from this allocation. From the point of view of period zero, there are $S$ possible outcomes associated with the allocation in period 1:

$$
V_{0}=\sum_{s} \pi_{s}\left(u\left(c_{s}\right)+\beta V_{s}\right) .
$$


Agent 1 must be better off choosing $c$ than opting not to make any transfers; hence:

$$
V_{0} \geqq \sum_{s} \pi_{s}\left(u\left(c_{s}^{\prime}\right)+\beta V_{s}^{\prime}\right)
$$

where $c_{s}^{\prime}$ and $V_{s}^{\prime}$ are his state $s$ consumption and continuation utilities respectively if he fails to make any transfers. But $c_{s}^{\prime} \geqq y_{s}$ and $V_{s}^{\prime} \geqq M$; this implies that:

$$
V_{0} \geqq \sum_{s} \pi_{s}\left(u\left(y_{s}\right)+\beta_{M}\right) .
$$

Since $V_{0}$ is any subgame-perfect utility level, it follows that:

$$
M \geqq\left(\sum_{s} \pi_{s}\left(u\left(y_{s}\right)\right) /(1-\beta) .\right.
$$

Since autarky is subgame perfect, the weak inequality is actually an equality.

Proof of Proposition 2.1. Suppose an allocation satisfies this condition. Write down a non-negative transfer scheme that generates the allocation (this is possible because it is feasible). Then specify the following strategy for each individual. If in the past, all individuals have acted so as to follow the transfer scheme, then follow it yourself today. If they have not, then make no transfers. This collection of strategies is clearly a subgame-perfect equilibrium.

Now suppose that the allocation $\left(c^{1}, c^{2}\right)$ is subgame perfect. Consider agent 1 at a particular date and state. We know that it must be at least as good for him to consume $c_{t}$ as to make no transfers; hence:

$$
u\left(c_{t}\right)+E_{t} \sum \beta^{\tau} u\left(c_{t+\tau}\right) \geqq u\left(c_{t}^{\prime}\right)+\beta V^{\prime}
$$

where $c_{r}^{\prime}$ is his current consumption and $V^{\prime}$ is his expected utility from future consumption if he makes no transfers today. Since $c_{t}^{\prime} \geqq y_{t}$, and $V^{\prime} \geqq V_{\text {aut }}$, (1) follows. \|

Proof of Proposition 3.1. I will prove the proposition for $k=1$; the other values of $k$ can be handled similarly. Note that the first-order conditions (1-2) imply that:

$$
\begin{aligned}
c & =f\left(y, Y_{t}, u_{t-1}\right) \\
& =f\left(y, Y_{t}, g\left(y_{t-1}, Y_{t-1}, u_{t-2}\right)\right)
\end{aligned}
$$

where $f$ and $g$ are both non-decreasing in all arguments. Hence:

$$
\operatorname{Cov}\left(c, y_{t-1} \mid y, Y_{t}, Y_{t-1}, u_{t-2}\right) \geqq 0 .
$$

Recall from basic probability that if $F$ is a finer sigma algebra than $G$, then:

$$
E\{\operatorname{Cov}(X, Y \mid F) \mid G\}=\operatorname{Cov}(X, Y \mid G)-\operatorname{Cov}\{E(X \mid F), E(Y \mid F) \mid G\} .
$$

In this case, think about $F$ as the sigma algebra generated by $\left(y, Y_{t}, Y_{t-1}, u_{t-2}\right)$, and $G$ as the sigma algebra generated by $Y_{t-1}$. Because $y_{-1}$ is independent of $\left(Y_{t}, y, u_{t-2}\right)$, we can conclude that:

$$
0 \leqq E\left\{\operatorname{Cov}\left(c, y_{t-1} \mid y, Y_{t}, Y_{t-1}, u_{t-2}\right) \mid Y_{t-1}\right\}=\operatorname{Cov}\left(c, y_{t-1} \mid Y_{t-1}\right)
$$

which concludes the proof of the inequality for $k=1$.

Finally, note that $\operatorname{Cov}\left(c, y \mid Y_{t}, u_{t-1}\right)=0$ for all values of $Y_{t}$ if and only if all $s$ lie in $S_{3}$ when $u_{0}=u_{t-1}$. But this means that $u_{s}=u_{t-1}$ for all $s$, and so neither agent's sustainability constraint will ever bind again.

Proof of Proposition 4.1. Consider the set of sample paths along which every state $s$ occurs infinitely often; this set of paths has measure one. Pick an arbitrary sample path in this set. We know that along this

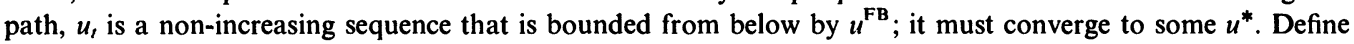
$v(u)=\operatorname{Min}_{s} u_{s}(u) ; v$ is continuous. Because every state occurs infinitely often along this path, for any $T$, there exists $t>T$ such that $u_{t}<v\left(u_{T}\right)$. Allowing $T$ to go to infinity, we see that $u^{*} \leqq v\left(u^{*}\right)$. But $u^{*}>v\left(u^{*}\right)$ for all

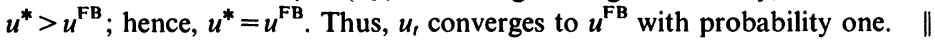

Proof of Proposition 4.2. $V$ is continuous and maps $\left[V_{\text {aut }}, V_{\max }\right]$ into itself; hence, $V$ has a fixed point $u^{*}$ such that $V\left(u^{*}\right)=u^{*}$. Thus, there is an efficient subgame-perfect allocation that provides both agents with the same level of utility. Suppose $S_{1}$ is empty when $u_{0}=u^{*}$. The symmetry of the problem then says that $S_{2}$ should be empty also. But this is impossible because there is no subgame-perfect first-best allocation. Thus, in the efficient allocation in which both agents receive utility $u^{*}, S_{1}$ and $S_{2}$ are both non-empty in period one.

I want to prove that starting from $u_{0}=V_{\text {aut }}$, there is some $t$ such that $u_{t}>u^{*}$ with positive probability. Define the function $v(u) \equiv \operatorname{Max}_{s} u_{s}(u)$. Start with an arbitrary $u_{0}<u^{*}$. We know that if $S_{1}$ is non-empty when $u_{0}=u^{*}, S_{1}$ is non-empty for any $u_{0} \in\left(V_{\text {aut }}, u^{*}\right)$; because there is a non-autarkic subgame-perfect allocation, if $u_{0}=V_{\text {aut }}$, there exists some $s$ such that $u_{s}>V_{\text {aut }}$. Hence, $v\left(u_{0}\right)>u_{0}$ for all $u_{0} \in\left[V_{\text {aut }}, u^{*}\right)$. Define the sequence $\left\{w_{n}\right\}_{t=1}^{N}$ recursively by the formula $w_{n}=v\left(w_{n-1}\right)$ and $w_{0}=u_{0}$. The set $S_{1}$ must be non-empty for any initial level of utility less than $u^{*}$ so $w_{n}>w_{n-1}$ for any $w_{n-1} \leqq u^{*}$. 
Suppose there does not exist any $n$ such that $w_{n}>u^{*}$. Then, $\left\{w_{n}\right\}_{n=1}^{N}$ is a strictly increasing sequence that is bounded from above by $u^{*} ; w_{n}$ converges to some limit $w^{*}$ that is less than or equal to $u^{*}$. Since $v$ is continuous (from the Theorem of the Maximum), this limit must satisfy $v\left(w^{*}\right)=w^{*}$. But this is impossible because $v(u)>u$ for any $u \leqq u^{*}$.

Thus, it is possible to start at $u_{0}=V_{\text {aut }}$ and to find $t$ such that the probability that $u_{t}$ exceeds $u^{*}$ is a positive value $\varepsilon$. Similarly, we can find $\tau$ such that if $u_{0}=V_{\max }, \operatorname{Pr}\left(u_{\tau}<u^{*} \mid u_{0}=V_{\max }\right)>\varepsilon$. The utility Markov process satisfies Assumption 12.1 of SLP and therefore Theorem 12.12 of SLP applies to it.

Note that the limiting distribution of utility must be symmetric. Hence, it can only be degenerate if it places all mass on $u^{*}$ such that $V\left(u^{*}\right)=u^{*}$; however, both agents' sustainability constraints bind with positive probability when $u_{0}=u^{*}$ (or it would be first best), and so a distribution with all mass on $u^{*}$ cannot be a stochastic steady-state. Hence, the steady-state cross-sectional distribution of utility is not degenerate in an efficient allocation. \|

Acknowledgements. Earlier versions of this paper were presented as "Efficient Bilateral Risk Sharing without Commitment", at the 1994 SEDC meetings, the 1994 MEDS Summer Macro Workshop, the University of Illinois, the University of Iowa, the University of Virginia, and the University of Wisconsin. I thank Dean Corbae, John Heaton, Hanan Jacoby, Barbara McCutcheon, Tom Pogue, Bart Taub, Cheng Wang, Steve Williamson, two anonymous referees and the editor, Ian Jewitt, for their comments. I acknowledge the support of NSF grant SES-92-23257. The opinions expressed in this paper are not necessarily those of the Federal Reserve Bank of Minneapolis or the Federal Reserve System.

\section{REFERENCES}

$\rightarrow$ ABREU, D. (1988), “On the Theory of Infinitely Repeated Games with Discounting”, Econometrica, 56, 383397.

ALVAREZ, F. and JERMANN, U. (1995), “Asset Pricing when Risk Sharing is Limited By Default” (University of Pennsylvania, mimeo).

ASHEIM, G. and STRAND, J. (1991), "Long-Term Union-Firm Contracts”, Journal of Economics, 53, 161184.

ATKESON, A. (1991), "International Lending With Moral Hazard and Risk of Repudiation", Econometrica, 59, $1069-1104$.

ATKESON, A. and LUCAS, R. (1992), “On Efficient Distribution with Private Information”, Review of Economic Studies, 59, 427-453.

$\rightarrow$ BEAUDRY, P. and DINARDO, J. (1995), "Is the Behavior of Hours Worked Consistent with Implicit Contract Theory?", Quarterly Journal of Economics, 110, 743-768.

BLUME, A. and CORBAE, D. (1994), "Enforcement and the Value of Money" (University of Iowa, mimeo).

COATE, S. and RAVAILLON, M. (1993), "Reciprocity Without Commitment: Characterization and Performance of Informal Insurance Arrangements", Journal of Development Economics, 40, 1-24.

COCHRANE, J. (1991), “A Simple Test of Consumption Insurance”, Journal of Political Economy, 99, 957976.

FARRELL, J. and MASKIN, E. (1989), "Renegotiation in Repeated Games", Games and Economic Behavior, $1,327-360$.

GAUTHIER, C. and POITEVIN, M. (1994), "Using Ex-ante Payments in Self-Enforcing Risk-Sharing Contracts" (Universite de Montreal, CRDE Working Paper No. 0394).

GREEN, E. (1987), "Lending and the Smoothing of Uninsurable Income", in E. Prescott and N. Wallace (eds.), Contractual Arrangements for Intertemporal Trade.

HALLER, H. (1990), "Games of Non-contractual Borrowing and Lending" (Virginia Polytechnic Institute, mimeo).

HART, O. and MOORE, J. (1989), "Default and Renegotiation: A Dynamic Model of Debt" (MIT, mimeo).

KIMBALL, M. (1988), "Farmers' Cooperatives as Behavior Toward Risk", American Economic Review, 78, 224-236.

KOCHERLAKOTA, N. (1994), "Efficient Bilateral Risk Sharing Without Commitment" (University of Iowa, mimeo).

LIGON, E., THOMAS, J. and WORRALL, T. (1996), "Informal Insurance Arrangements in Village Economies" (University of Warwick, mimeo).

MARCET, A. and MARIMON, R. (1992), "Communication, Commitment, and Growth", Journal of Economic Theory, 58, 219-249.

MARCET, A. and MARIMON, R. (1995), "Recursive Contracts" (Universitat Pompeu Fabra, mimeo).

PHELAN, C. (1995), "Repeated Moral Hazard and One-Sided Commitment", Journal of Economic Theory, 66, 488-506.

PHELAN, C. and TOWNSEND, R. (1991), "Computing Multiperiod Information-Constrained Optima", Review of Economic Studies, 58, 853-881.

SPEAR, S. and SRIVASTAVA, S. (1987), "On Repeated Moral Hazard with Discounting", Review of Economic Studies, 54, 599-617. 
STOKEY, N., LUCAS, R. and PRESCOTT, E. (1989) Recursive Methods in Economic Dynamics (Cambridge, MA.: Harvard University Press).

TAUB, B. (1989), "Dynamic Consistency of Insurance Contracts Under Enforcement by Exclusion", Journal of Economic Dynamics and Control, 13, 93-112.

THOMAS, J. and WORRALL, T. (1988), "Self-Enforcing Wage Contracts", Review of Economic Studies, 55, $541-554$.

THOMAS, J. and WORRALL, T. (1990), "Income Fluctuations and Asymmetric Information: An Example of the Repeated Principal-Agent Problem", Journal of Economic Theory, 51, 367-390.

TOWNSEND, R. (1987), "Arrow-Debreu Programs as Microfoundations of Macroeconomics", in T. Bewley (ed.), Advances in Economic Theory: Fifth World Congress (Cambridge, UK.: Cambridge University Press).

TOWNSEND, R. (1994), "Risk and Insurance in Village India", Econometrica, 62, 539-592.

WANG, C. (1995), "Dynamic Insurance with Private Information and Balanced Budgets", Review of Economic Studies, 62, 577-596.

ZHANG, H. (1993), “An Asset Market with an Endogenous Rationality Constraint” (Duke University, mimeo). 